\title{
ACEPTACIÓN Y FUNCIONAMIENTO DEL DERECHO
}

\section{RESUMEN}

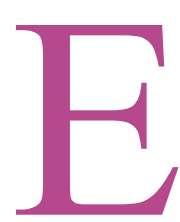

1 positivismo jurídico sostiene que el derecho es una técnica de motivación de comportamientos y que la existencia del derecho depende de hechos sociales. En este trabajo nos proponemos analizar dos aspectos de estos problemas:

a) la relación entre las normas jurídicas y las razones para la acción. Si las normas jurídicas intervienen en el razonamiento práctico de los sujetos y determinan sus acciones, entonces el derecho guía la conducta de los sujetos. En estos casos, el derecho es «efectivo»;

b) la relación entre los criterios de existencia y efectividad del derecho. Siguiendo a Hart, la afirmación acerca de la existencia de un sistema jurídico $\mathrm{Sj}$ es un enunciado bifronte respecto de la eficacia y la aceptación de las normas de $\mathrm{Sj}$. También es frecuente afirmar que $\mathrm{Sj}$ guía la conducta de los sujetos si y sólo si es eficaz. Estas consideraciones sugieren que los sistemas jurídicos, por el mero hecho de existir, guían el comportamiento. Sin embargo, esto supone una confusión entre «eficacia» y «efectividad». Al distinguirse ambos conceptos se advierte la necesidad de establecer criterios para comprobar si las normas jurídicas funcionan como razones para la acción. Para esclarecer este punto, se proponen dos criterios de efectividad del derecho y, se muestran las dificultades que supone utilizar a la aceptación de las normas como criterio de existencia y efectividad del derecho.

\section{Introducción}

1. Se sostiene, a menudo, que el derecho es una técnica de motivación de comportamientos. La existencia de las normas jurídicas 
tiene por objeto, al menos, afectar el comportamiento de los sujetos para dar lugar a estados de cosas deseados por la autoridad normativa. Por medio de prescripciones, la autoridad normativa intenta motivar la conducta de los sujetos ${ }^{1}$. Es evidente que las autoridades pueden fracasar en sus intentos; entonces, el éxito en la motivación de los sujetos puede considerarse como un criterio de la habilidad de las autoridades respecto del uso de un instrumento. Si denominamos «efectividad» a la motivación de las conductas, puede considerarse más hábil (capaz) a aquella autoridad cuyas prescripciones sean más efectivas. Por tanto, desde un punto de vista instrumental, la efectividad de las normas de un sistema puede ser considerado como criterio de evaluación de la calidad técnica de los sistemas jurídicos. Obviamente, un sistema puede ser evaluado con criterios alternativos y, más aún, la distinción entre diferentes tipos de cualidades posibilita analizar las relaciones entre la calidad instrumental del derecho y otras de la bondad ${ }^{2}$.

2. Supóngase que la autoridad normativa prescribe la acción p. Esto implica que intenta que los sujetos hagan p. Sin embargo, los sujetos pueden negarle (o simplemente no advertir) el carácter normativo a los enunciados de la autoridad normativa. En el caso de las normas jurídicas, el reconocimiento de la intención de obligar a comportarse de una manera específica es una condición necesaria de la efectividad del derecho ${ }^{3}$. A partir del reconocimiento de la naturaleza prescriptiva de la expresión de la autoridad normativa, se pueden originar distintas situaciones relevantes para el análisis del razonamiento práctico de los sujetos:

a) el sujeto considera que las normas no poseen suficiente importancia al momento de decidir un curso de comportamiento y no ejecuta la acción prescripta,

b) el sujeto ejecuta la acción prescripta, pero su comportamiento no está motivado por la norma jurídica sino por hábito,

${ }^{1}$ Cfr. von Wright, Georg, «Norma y acción: una investigación lógica» (trad. de Pedro García), pág. 133, Tecnos, 1970, Madrid, «Norms, Truth and Logic», en Practical Reason, págs. 138, 139, Cornell UP, 1983, Ithaca. Londres.

${ }^{2}$ Cfr. von Wright, Georg, «The Varieties of Goodness», Routledge \& Kegan Paul, 1963,

${ }^{3}$ Cfr. Ross, Alf, «Directives and Norms», pág. 39, Routledge \& Kegan Paul, 1968, Londres. Alchourrón, Carlos, y Bulygin, Eugenio, «Von Wright on Deontic Logic and The Philosophy of Law», en The PhiIosophy of Georg Henrik von Wright. P. Schilpp y L. Hahn (eds.), pág. 668, La Salle, Illinois, 1989. Von Wright, Georg, «0n Promise», en Practical Reason, op. cit., pág. 84. 
actitud de imitación, razones morales, etc. Denominaremos a esta situación «conformidad al derecho»,

c) el sujeto efectúa la acción prescripta en virtud de que es prescripta por el derecho. Denominaremos a esta situación «cumplimiento del derecho» ${ }^{4}$.

3. En los casos de conformidad al derecho y de cumplimiento del derecho es frecuente afirmar que el sistema jurídico es eficaz. A su vez, es usual señalar que el derecho guía el comportamiento si y sólo si es eficaz 5 . Sin embargo, un sistema puede ser eficaz en el sentido de conformidad al derecho y las normas no intervenir en el razonamiento práctico de los sujetos. Por consiguiente, aun en el supuesto de la eficacia del sistema jurídico, es pertinente preguntar lo siguiente: ¿las normas guían el comportamiento de los sujetos? Si esta expresión significa que las normas jurídicas intervienen en el razonamiento práctico de los sujetos y determinan sus acciones, entonces, la eficacia del derecho no es un criterio suficiente para responder afirmativamente. Por tanto, a los efectos de clarificar el papel de las normas en el razonamiento de los sujetos, analizaremos a las normas jurídicas desde la perspectiva de las razones para la acción.

\section{Normas y razones para la acción}

1. Si las normas son consideradas como un instrumento utilizado por las autoridades para determinar las acciones de los sujetos es obvio que las autoridades intentan que las normas constituyan razones para la acción. Siguiendo a G. von Wright, podemos clasificar a las razones para la acción en ${ }^{6}$ :

a) razones internas: son una combinación de actitudes volitivas (intención de conseguir un determinado fin) y actitudes epistémicas

${ }^{4}$ Kelsen, Hans, «Teoría pura del derecho», 2. ${ }^{a}$ edición (trad. de Roberto Vernengo), págs. 40-41, UNAM, 1979, México, Raz, Joseph, «Rethinking Exclusionary Reason» en Practical Reason and Norms, 2. a edición, pág. 179. Hutehinson, 1990, Londres. Navarro, Pablo, «La eficacia del Derecho». pág. 16, Centro de Estudios Constitucionales, 1990, Madrid.

${ }^{5}$ Raz, Joseph: «Postscripturn», en El concepto de sistema jurídico, 2. ${ }^{\mathrm{a}}$ edición (trad. de R. Tamayo), págs. 276 y sigs., UNAM, 1986, México. UNAM, Peter, «Efectiveness», en ARSP, pág. 484 (1983).

${ }^{6}$ Von Wright, Georg, «Explanation and Understandig ofHuman Action». en Practical Reason, op. cit., pág. 54. «Freedom and Determination», págs. 28 y sigs. Acta Philosophica Fennica, vol. XXXI, North HolandPC. 1980, Amsterdam. 
(creencia acerca de los medios necesarios para obtener el fin perseguido). Las razones de este tipo son necesariamente razones del sujeto para actuar, aun cuando pueden ser derrotadas por razones en contra de la acción.

b) razones externas: son pautas o estímulos simbólicos que los sujetos aprenden a reconocer y responder en conformidad. La presentación y respuesta a los estímulos simbólicos son «acciones comunicativas» y consisten en participar en una práctica institucionalizada. Las órdenes, pedidos, preguntas, normas y señales son ejemplos de razones externas. Este tipo de razones son razones contingentes para la acción.

Esta distinción permite destacar las siguientes alternativas de vinculación entre normas, razones y acciones:

a) el sujeto identifica una norma y, sin embargo, no se encuentra motivado por ella. La norma no constituye una razón del sujeto, aun cuando, desde un punto de vista externo, la norma existe como razón para la acción en relación a determinado grupo social;

b) el sujeto procura satisfacer ciertos deseos, intereses y objetivos. Estos pueden ser de diferentes tipos: i) evitar la sanción y sus consecuencias, ii) promover un estado de cosas coincidente con los fines establecidos respecto de una norma, iii) cumplir con las exigencias de su rol en la sociedad, ete. En estos casos, la norma incide en el comportamiento del sujeto pero no constituye una razón externa del sujeto. La conducta del sujeto conforme a la norma responde a razones internas que determinan la acción con independencia del estímulo provisto por la norma, es decir: la acción del sujeto no es intención bajo (a tenor de) la descripción «cumplimiento de la norma» ${ }^{7}$. El conocimiento de la norma no determina la intención de actuar del sujeto sino que constituye un criterio para seleccionar entre acciones posibles a los efectos de alcanzar un estado de cosas que el sujeto persigue con independencia de las normas jurídicas;

c) el sujeto acepta la norma y está dispuesto a seguirla con independencia de la presión que ejerce la sanción o de la coincidencia

${ }^{7}$ Cfr. Davidson, Donald: «Acciones, razones y causas», en La filosofía de la acción humana, White, A. (ed.), 1976, Fondo de Cultura Económica, México. Von Wright, Georg, «On the Logic of Norms and Actions», en Practical Reason, op. cit., pág. 115. Acerca del aspecto pragmático de los criterios utilizados para individualizar acciones, cfr. Guibourg, Ricardo, «Algunas reflexiones acerca del concepto de acción humana», en El Fenómeno Normativo, Astrea, 1987, Buenos Aires. 
entre sus objetivos y los fines asignados a la norma. En este caso es usual señalar que el sujeto ha internalizado las normas ${ }^{8}$.

2. Algunos teóricos han sugerido que es plausible definir a las normas en relación a las razones para la acción, y que constituye un serio defecto no exhibir las vinculaciones entre la normatividad del derecho y las razones para actuar'. Sin embargo, es necesario advertir que la existencia de una norma, en tanto que razón para actuar, no puede depender del reconocimiento de los sujetos, en todos los casos concretos, de su carácter de razón ${ }^{10}$. Por tanto, la existencia de una norma como razón tiene que diferenciarse de su funcionamiento como tal en las consideraciones del sujeto. Esto permite sugerir que la vinculación entre normas y razones para la acción no constituye un criterio de existencia de normas o sistemas normativos, sino una pauta de evaluación de su incidencia en la conducta de los sujetos.

En resumen: una norma puede incidir en el comportamiento de un sujeto de las siguientes maneras:

i) en virtud de su aceptación o internalización,

ii) como razón externa.

iii) como información relevante para la persecución de fines personales del sujeto.

\section{Aceptación del Derecho}

1. Herbert Hart sostiene que los enunciados acerca de la existencia de un sistema jurídico son bifrontes ${ }^{11}$. Por una parte, el sistema tiene que ser eficaz y por otra parte, los funcionarios tienen que aceptar, al menos, las reglas secundarias. Según señalamos anteriormente, la eficacia es una condición necesaria de la efectividad, aunque no es obvio como se relacionan los criterios de efectividad con los criterios de existencia de los sistemas 39.

${ }^{8}$ Von Wright, Georg: «Determinism and the Study of Man», en Practical Reason, op. cit., pág.

${ }^{9}$ Raz, Joseph, «Las razones de las acciones, decisiones y normas», en Razonamiento práctico, Raz J. (ed.) (trad. de J. Utrilla), 1986. Fondo de Cultura Económica, México. Nino, Carlos, «El concepto de validez en la teoría de Kelsen», en La validez del Derecho, 1985, Astrea, Bs As.

Doxa.

${ }^{10}$ Navarro, Pablo, y Redondo, Cristina, «Normas y razonamiento práctico», a publicarse en

${ }^{11}$ Hart, Herbert, «El concepto de derecho» (trad. de Genaro Carrió). pág. 145, Abeledo-Perrot, 1968, Buenos Aires. 
jurídicos. 
Si discriminamos entre eficacia y aceptación del derecho como condiciones de existencia de un sistema, hay que recalcar que la mera conformidad entre normas y conductas no proporciona información sobre las motivaciones de los sujetos. Por tanto, descartada la eficacia, es conveniente analizar la relación entre aceptación y funcionamiento del derecho.

2. La aceptación de una norma presupone: i) el sujeto conoce lo prescriptivo por la norma y ii) el sujeto tiene una actitud positiva respecto de la norma ${ }^{12}$. La aceptación de una norma se constituye en una razón del sujeto para comportarse de una manera específica. En este sentido, la aceptación es una razón interna para la acción, aunque puede resultar vencida por razones alternativas de mayor peso.

Sin embargo, la expresión «aceptación de las normas» es ambigua, ya que oculta diferencias relevantes entre las actitudes de los sujetos y los objetos a los que se dirige. Respecto de las actitudes, la aceptación puede manifestarse en ${ }^{13}$ :

a) una disposición a la acción,

b) una actitud crítica respecto de la desviación de la pauta prescripta,

c) admitir como justificada la crítica por la desviación, propia o de otro sujeto, respecto de la pauta prescripta,

d) el uso del lenguaje normativo para evaluar una pauta de comportamiento.

En el presente trabajo tendremos en cuenta solamente a los destinatarios de las normas; $y$, en este sentido, la aceptación de una norma implica una disposición a la acción prescripta $^{14}$. Sin embargo, la disposición a la acción puede presentar distintos grados de intensidad según sean las razones del sujeto para aceptar las normas. Por tanto, es necesario distinguir cuidadosamente, al momento de evaluar la efectividad del derecho, entre ${ }^{15}$ : i) las razones para aceptar una norma y ii) el hecho de que la aceptación

${ }^{12}$ Nino, Carlos, «El concepto de Derecho de Hart», en H. L. A. Hart y el concepto de derecho, A. Squella (ed.), pág. 51, 1986, Facultad de Ciencias Jurídicas de la Universidad de Valparaíso, Chile. MacCormick, Neil, «Reglas sociales», en H. L. A. Harty el Concepto de Derecho, op. cit., págs. 304 y sigs.

${ }^{13}$ Haecker, P. M., «Hart's Philosophy of Law», en Law, Morality, and Society. Essays in Honour of H. L, A. Hart, Haecker, P. y Raz, J. (eds.), págs. 1-25, 1977. Clarendon Press, Oxford. Barcelona.

${ }^{14}$ Kliemt, Hartmut, Instituciones morales (trad. de Jorge Malem Seña), pág. 170, Alfa, 1987,

${ }^{15}$ Kliemt, Harmut, Instituciones morales, op. cit., pág. 187. 
de una norma constituya una razón del sujeto para actuar.

3. La aceptación de las normas jurídicas sirve para analizar la actitud de los sujetos respecto de diferentes objetos:

a) puede interpretarse como la disposición o consentimiento a evaluar la conducta humana desde una perspectiva normativa. En este sentido, frecuentemente, enfatizamos que un anarquista no acepta (rechaza) el derecho o el estado. La importancia de este aspecto es indirecta respecto de los problemas de efectividad del derecho y, por ello, no será objeto de mayor consideración en este trabajo;

b) los sujetos aprueban el contenido normativo de determinadas prescripciones, es decir: los sujetos creen que el contenido normativo es una pauta de comportamiento correcta o útil ${ }^{16}$;

c) los sujetos reconocen (aprueban, consideran) que, en determinados ámbitos, deben guiar su conducta por medio de normas jurídicas. En otras palabras, los sujetos consideran imprescindible la regulación de ciertas esferas del comportamiento. Puede ser que no estén satisfechos o discrepen acerca del contenido específico de la regulación, pero reconocen la necesidad y aprueban la existencia de tal regulación. También puede suceder que los sujetos aceptan las normas superiores del sistema y en virtud de ello, admiten que deben comportarse de acuerdo a las normas derivadas del sistema y de acuerdo con las normas creadas por las autoridades a las que las normas superiores otorgan competencia. A los fines de simplificar la exposición, diremos que, en estos casos, los sujetos aceptan la existencia institucional de las normas ${ }^{17}$.

\section{Criterios de efectividad}

1. Al intentar formular un criterio de efectividad de un sistema jurídico $\mathrm{Sj}$ presupondremos que $\mathrm{Sj}$ es eficaz. De este modo, la pregunta respecto de la efectividad de $\mathrm{Sj}$ es un interrogante acerca de las razones de la conducta conforme a las normas de Si. Afirmar verdaderamente que $\mathrm{Sj}$ es efectivo significa que, en la explicación de las acciones de los sujetos, la descripción de las normas de $\mathrm{Sj}$ no constituyen premisas superfluas sino que por el contrario, son determinantes del comportamiento de los sujetos.

${ }^{16}$ Hart, Herbert, El concepto de Derecho, op. cit. pág. 251.

${ }^{17}$ Kliemt, Harmut, Instituciones morales, op. cit., pág. 195. 
2. La aceptación de una norma es una relación entre la actitud positiva de los sujetos y las normas. Esta relación puede ser la aceptación del contenido prescripto, o bien, la aceptación de la existencia institucional de las normas. La aceptación de una norma se modifica al modificarse los miembros de la relación: cuando se altera la actitud de los sujetos o cuando cambia el objeto al que se dirige.

Con la ayuda de estos conceptos, puede enunciarse el siguiente criterio de efectividad para un sistema $\mathrm{Sj}$ :

$\mathrm{C} 1$ : En un tiempo T, $\mathrm{Sj}$ es efectivo si y sólo si los destinatarios aceptan el contenido normativo de las normas de $\mathrm{Sj}$, y esta actitud es la razón de la conducta conforme de los sujetos.

Un sistema eficaz podría considerarse efectivo si satisface $\mathrm{C} 1$. La aplicación de un criterio de efectividad de normas generales se enfrenta con problemas de vaguedad. La asignación de la propiedad «efectividad» a una norma general puede suscitar discusión en razón de que no es posible justificar concluyentemente un límite para el uso del concepto. También, al igual que la eficacia, la aplicación de los criterios de efectividad puede limitarse a «casos relevantes», por ejemplo: aquellos casos en los que se pone a prueba la identidad del sistema ${ }^{18}$.

3. Este criterio de efectividad es relativo al consenso de los sujetos de un grupo respecto del contenido del derecho; es decir, el sistema es efectivo si y sólo si la conformidad se basa en la adhesión de los sujetos a las prescripciones del mismo (en razón de su justicia, utilidad, etc.). Sin embargo, es posible que existan sistemas jurídicos con alto poder motivador, sin que ello suponga la adhesión a sus contenidos normativos. Si bien la justicia de las instituciones y prescripciones jurídicas puede influir en la eficacia del derecho es necesario advertir que un derecho considerado injusto por los sujetos puede ser técnicamente excelente al motivar el comportamiento y, también, un sistema aceptado por los sujetos en virtud de la justicia de sus prescripciones puede ser inefectivo porque los sujetos pueden obrar únicamente en virtud de sus convicciones morales. Por consiguiente, la legitimación de un sistema jurídico, entendida como aceptación de sus contenidos normativos, no es condición suficiente ni necesaria de su efectividad. Sin embargo, hay que destacar que esto no significa negar que la aceptación y la eficacia de las normas sean condiciones necesarias y suficientes de la existencia de los sistemas jurídicos. Probablemente, uno de los defectos más serios de $\mathrm{C} 1$ es que dificulta el análisis del impacto de las normas jurídicas en

\footnotetext{
${ }^{18}$ Navarro, Pablo, La eficacia del derecho, op. cit., pág.
} 
la conducta de los sujetos. Esto se debe a que, normalmente, la moral, la religión y el derecho intersectan en la regulación de las conductas. Por tanto, puede ser que las actitudes de los sujetos se dirijan hacia el sistema moral del grupo y su conformidad con el contenido del derecho sea una simple coincidencia ${ }^{19}$.

4. La aceptación del derecho puede referirse a un objeto diferente del contenido normativo: la existencia institucional de las normas. Aceptar la existencia institucional de una norma se manifiesta en una disposición a obedecer la norma de igual modo en que aceptar un contenido normativo se manifiesta en una disposición a ejecutar la acción prescripta. En ambos casos, la disposición a obedecer o a ejecutar la acción puede estar en conflicto con otras razones del sujeto.

Es importante señalar que, si bien la aceptación de los contenidos no es necesario ni suficiente del éxito en la motivación de conductas; por el contrario, la aceptación de la existencia de la regla es imprescindible a los efectos de verificar si el derecho es técnicamente bueno. Conforme a ello, puede enunciarse el siguiente criterio:

C2: En un tiempo $\mathrm{T}$, un sistema jurídico $\mathrm{Sj}$ es efectivo si y sólo si los sujetos aceptan la existencia institucional de las normas de $\mathrm{Sj}$, y esta es la razón de la conducta conforme de los sujetos.

Podría argumentarse que $\mathrm{C} 2$ presupone a $\mathrm{C} 1$, porque la aceptación de la existencia de la norma implicaría la aceptación de su contenido, en virtud de que quien está dispuesto a obedecer una norma está dispuesto a ejecutar el contenido prescripto por ella. Sin embargo, es preciso advertir que el sujeto puede tener una opinión negativa respecto del contenido de la norma y, pese a ello, sentirse inclinado a realizar la acción en razón de su disposición a seguir la norma. Por tanto, aunque la relación entre obedecer una norma y ejecutar su contenido es analítica, la vinculación entre aceptar obedecer una norma y aceptar el contenido normativo es de índole diferente. Una actitud positiva hacia la existencia de una norma implica una disposición a la acción, pero una disposición a la acción no implica una actitud positiva hacia la misma.

${ }^{19}$ Nino, Carlos. «La validez de las normas de facto», en La validez del derecho, op. cit., pág. 95. Kliemt, Harmut. Instituciones morales, op.cit., pág. 171. Lagerspotz, Erik, «Hart and the Separation Thesis», en ARSP, LXXV heft 2, pág. 244, 1989. 
$\triangle$

DOXA-9 (1991) 